\title{
Placement Sensitivity to Aberration in Optical Imaging
}

\author{
Giuseppe Y. H. Mak, Edmund Y. Lam and Alfred K. Wong
}

\begin{abstract}
Theories are developed to quantify the shift of image intensity extremum $(\Delta x)$ due to aberration. The theory on real, one-dimensional mask spectrum has been extended to complex, two-dimensional mask spectrum under coherent and partially coherent imaging. Verification of the formulae was performed on alternating phase-shifting mask (PSM) and contact array. Balanced third-order coma was used to illustrate the validity of the theories. It is found that the image shift due to aberration of alternating PSM decreases when the partial coherence factor increases. In general, the theories can be applied to any mask spectra and aberration functions.
\end{abstract}

\section{INTRODUCTION}

Due to the continuous decrease of critical dimension (CD) and the increase of integration density in integrated circuits (ICs), image placement error (or simply placement error) is becoming an important source of error in lithographic applications, such as the 1G DRAM development processes $(0.18 \mu \mathrm{m}$ design rules) [1]. This error is normally quantified by the image center shift of a feature, e.g. a line or a contact. If image skew is negligible, extremum shift (the point where maximum or minimum intensity occurs) can be used to represent image placement error [2].

Aberration arises from the optical path difference of light rays that pass through the exit pupil of an exposure system. This phenomenon causes a range of problems in photolithography. It has been reported that the depth of focus (DOF) of semi-randomly aligned patterns with altemating PSMs decreases due to spherical aberration [3] Other kinds of aberration can cause imaging artifacts such as distortion and asymmetry [4]. In this paper, we focus on the effect of aberration towards the shift of image intensity extremum.

At present, theories have been developed for calculating the image placement error when the photomask has a real, one-dimensional (1D) mask spectrum under coherent imaging [2]. However, since the geometric patterns on a photomask are arbitrary, we aim at developing formulae that are applicable to complex, two-dimensional (2D) mask spectra.

In the next section, formulae for image shifts are derived. Three cases are considered:

Giuseppe Y. H. Mak and Edmund Y. Lam are with the Department of Electrical and Electronic Engineering, the University of Hong Kong, Hong Kong. Alfred K. Wong is now with the Fortis Systems, Inc., U.S.A. E-mail: yhgmak@eee.hku.hk, elam@eee.hku.hk. awong@fortis-systems.com.
1. 1D mask spectrum under coherent imaging

2. 1D mask spectrum under partially coherent imaging

3. 2D mask spectrum under coherent imaging.

Comparison is made between results from the formulae and those from aerial image simulation.

\section{THEORY}

\section{A. ID mask spectrum under coherent imaging}

We begin our analysis by considering the image intensity under coherent imaging [5]. Let $(\hat{x}, \hat{y})$ be the spatial coordinates normalized by $(\lambda / \mathrm{NA})$, where $\lambda$ is the wavelength and NA the numerical aperture. Also let $(\hat{f}, \hat{g})$ be the frequency coordinates normalized by $(N A / \lambda$ If the phase aberration is $\Phi(\hat{f}, \hat{g})$ and the ID mask spectrum is $\tilde{\hat{O}}_{x}(\hat{f})^{1}$, the image intensity $I$ is given by:

$$
\begin{aligned}
I(\hat{x}) & =\int_{-1-1}^{1} \int_{\hat{o}}^{\hat{o}_{x}}\left(\hat{f}_{1}\right) \tilde{\hat{o}}_{x}^{*}\left(\hat{f}_{2}\right) \\
& \cdot \exp \left\{-i 2 \pi\left[\left(\hat{f}_{1}-\hat{f}_{2}\right) \hat{x}+\left(\phi_{1}-\phi_{2}\right)\right]\right] d \hat{f}_{1} d \hat{f}_{2}
\end{aligned}
$$

where the asterisk denotes complex conjugation and $\phi_{i}=\Phi\left(\hat{f}_{i}, 0\right)$, for $i=1,2$. This integral can be written as:

$$
\begin{aligned}
I(\hat{x})= & \int_{-1}^{1}|\hat{\hat{o}}(\hat{f})| \hat{f}+2 \operatorname{Re}\left\{\int_{-1}^{1} \int_{\hat{f}_{2}}^{1} \tilde{\hat{o}}_{x}\left(\hat{f_{1}}\right) \tilde{\hat{o}}_{x}^{*}\left(\hat{f}_{2}\right)\right. \\
& \cdot \exp \left\{-i 2 \pi\left[\Delta \hat{f}_{12} \hat{x}+\Delta \phi_{12}\right] d \hat{f}_{1} d \hat{f}_{2}\right\}
\end{aligned}
$$

where $\operatorname{Re}($.$) denotes the real part of (.), \Delta \hat{f}_{12}=\hat{f}_{1}-\hat{f}_{2}$ and $\Delta \phi_{12}=\phi_{1}-\phi_{2}$. The intensity extremum is found by differentiating $I$ with respect to $\hat{x}$ :

$$
\begin{aligned}
\frac{d I}{d \hat{x}} & =4 \pi \int_{-1}^{1} \int_{\hat{f}_{2}}^{1} \operatorname{Im}\left\{\tilde{\hat{o}}_{x}\left(\hat{f}_{1}\right) \tilde{\hat{o}}_{x}^{*}\left(\hat{f}_{2}\right)\right. \\
& \left.\cdot \exp \left[-i 2 \pi\left(\Delta f_{12} \hat{x}+\Delta \phi_{12}\right)\right] d \hat{f}_{1} d \hat{f}_{2}\right\}
\end{aligned}
$$

where $\operatorname{Im}($.$) denotes the imaginary part of (.). By$ expanding the integrand $\operatorname{Im}($.$) of Eq. (5) into Taylor series$

\footnotetext{
${ }^{1}$ The superscript " $"$ means that the mask spectrum is obtained from the 2D Fourier transform of the mask transmission function $\hat{O}_{x}(\hat{x})$
} 
and retaining the first-order terms of the expansion, we have:

$$
\begin{aligned}
& \operatorname{Im}\{\}=D_{12}\left[\cos \left(2 \pi \Delta \phi_{12}\right)-2 \pi \Delta f_{12} \sin \left(2 \pi \Delta \phi_{12}\right) \hat{x}\right] \\
& -S_{12}\left[\sin \left(2 \pi \Delta \phi_{12}\right)+2 \pi \Delta f_{12} \cos \left(2 \pi \Delta \phi_{12}\right) \hat{x}\right] \\
& \text { where } \\
& D_{12}=\operatorname{Im} \tilde{\hat{o}}\left(\hat{f}_{1}\right) \operatorname{Re} \tilde{\hat{o}}\left(\hat{f}_{2}\right)-\operatorname{Re} \tilde{\hat{o}}\left(\hat{f}_{1}\right) \operatorname{Im} \tilde{\hat{o}}\left(\hat{f}_{2}\right) \\
& S_{12}=\operatorname{Re} \tilde{\hat{o}}\left(\hat{f}_{1}\right) \operatorname{Re} \tilde{\hat{o}}\left(\hat{f}_{2}\right)+\operatorname{Im} \tilde{\hat{o}}\left(\hat{f}_{1}\right) \operatorname{Im} \tilde{\hat{o}}\left(\hat{f}_{2}\right)
\end{aligned}
$$

Substituting Eq. (6) into Eq. (5) and setting $d I / d \hat{x}=0$, Eq (5) becomes a linear equation in $\hat{x}$. Solving for $\hat{x}$ :

$$
\Delta \hat{x}_{c}=\frac{\int_{-1}^{1} \int_{\hat{f}_{2}}^{1} \Delta f_{12}\left[D_{12} \cos \left(2 \pi \Delta \phi_{12}\right)-S_{12} \sin \left(2 \pi \Delta \phi_{12}\right)\right] d \hat{y}_{1} \hat{d}_{2}}{2 \pi \int_{-1}^{1} \int_{\hat{y}_{2}}^{1} \Delta S_{12}^{2}\left[D_{12} \cos \left(2 \pi \Delta \phi_{12}\right)+S_{12} \sin \left(2 \pi \Delta \phi_{12}\right)\right] \hat{d \hat{f}_{1}} \hat{d}_{2}}
$$

We assume that without aberration, the extremum exists at $\hat{x}=0$. Hence Eq. (7) represents the image shift due to aberration.

\section{B. ID mask spectrum under partially coherent imaging}

In partially coherent imaging, the effective light source consists of a distribution of point sources in the pupil plane [6]. Each point source produces an image that adds up to form the final image. If $J\left(\hat{f}_{s}, \hat{g}_{s}\right)$ represents the light source, the image intensity is given by:

$$
I(\hat{x})=\frac{\iint_{R} J\left(\hat{f}_{s}, \hat{g}_{s}\right) I_{s}\left(\hat{f}_{s} ; \hat{x}\right) d \hat{f}_{s} d \hat{g}_{s}}{\iint_{R} J\left(\hat{f}_{s}, \hat{g}_{s}\right) d \hat{f}_{s} d \hat{g}_{s}}
$$

where

$$
\begin{aligned}
I_{s}\left(\hat{f}_{s} ; \hat{x}\right)= & \int_{-1-1}^{1} \int_{\hat{o}_{x}}^{1} \tilde{\hat{f}}_{x}\left(\hat{f}_{1}-\hat{f}_{s}\right) \tilde{\hat{b}}_{x} \cdot\left(\hat{f}_{2}-\hat{f}_{s}\right) \\
& \cdot \exp \left(-i 2 \pi\left[\Delta \hat{f}_{12} \hat{x}+\Delta \phi_{12}\right] d \hat{f}_{1} d \hat{f}_{2}\right.
\end{aligned}
$$

is the image intensity due to the point source at $\left(\hat{f}_{s}, \hat{g}_{s}\right)$ and $\mathrm{R}$ is the extent of the light source. By setting $d I / d \hat{x}=0$, we have:

$$
\iint_{R} J\left(\hat{f}_{s}, \hat{g}_{s}\right)\left(d I_{s} / d \hat{x}\right) d \hat{f}_{s} d \hat{g}_{s}=0
$$

After some manipulation, the image shift is obtained as:

$$
\Delta \hat{x}_{p c}=\frac{\iint_{R} J\left(\hat{f}_{s}, \hat{g}_{s}\right) M\left(\hat{f}_{s}\right) d \hat{f}_{s} d \hat{g}_{s}}{\iint_{R} J\left(\hat{f}_{s}, \hat{g}_{s}\right) N\left(\hat{f}_{s}\right) d \hat{f}_{s} d \hat{g}_{s}}
$$

where $M$ and $N$ are the numerator and denominator in Eq. (7) respectively, but with the mask spectrum shifted.

\section{C. $2 D$ mask spectrum under coherent imaging}

Similar to Section II A, we consider the image intensity first:

$I(\hat{x}, \hat{y})=\mid \iint_{P} \tilde{\hat{o}}(\hat{f}, \hat{g}) \exp \left(\left.j 2 \pi[\hat{\hat{x}}+\hat{g} \hat{y}+\Phi(\hat{f}, \hat{g})] d \hat{f} d \hat{g}\right|^{2}\right.$

where $\mathrm{P}$ is the unit circle on the $\hat{f}$ - $\hat{\mathrm{g}}$ plane. Usually, the mask spectrum can be expressed as a weighted sum of delta functions:

$$
\tilde{o}(\hat{f}, \hat{g})=\sum_{k=1}^{N} a_{k} \delta\left(\hat{f}-\hat{f}_{k}, \hat{g}-\hat{g}_{k}\right)
$$

where $a_{k}$ is complex, $\sqrt{\hat{f}_{k}^{2}+\hat{g}_{k}^{2}} \leq 1$. Substituting Eq. (13) into Eq. (12), Eq. (12) becomes:

$$
\begin{aligned}
I(\hat{x}, \hat{y})= & \sum_{k=1}^{N}\left|a_{k}\right|^{2}+2 \operatorname{Re}\left\{\sum_{\substack{p<q \\
1 \leq p, q \leq N}} a_{p} a_{q}\right. \\
& \cdot \exp \left[-j 2 \pi\left(\Delta \hat{f}_{p q} \hat{x}+\Delta \hat{g}_{p q} \hat{y}+\Delta \phi_{p q}\right)\right\}
\end{aligned}
$$

To find the position of the extremum, Eq. (14) is partially differentiated with respect to $\hat{x}$ and $\hat{y}$. We want to find a pair of coordinates such that both partial derivatives equate to zero. For each partial derivative, the manipulations are similar to those in Section II A. Using first-order approximation, two linear equations in $\hat{x}$ and $\hat{y}$ result:

$$
\left\{\begin{array}{l}
A \hat{x}+B \hat{y}=C \\
B \hat{x}+D \hat{y}=E
\end{array}\right.
$$

where

$$
\begin{aligned}
& D_{p q}=\operatorname{Im} a_{p} \operatorname{Re} a_{q}-\operatorname{Re} a_{p} \operatorname{Im} a_{q}, \\
& S_{p q}=\operatorname{Re} a_{p} \operatorname{Re} a_{q}+\operatorname{Im} a_{p} \operatorname{Im} a_{q} \\
& U=D_{p q} \sin \left(2 \pi \Delta \phi_{p q}\right)+S_{p q} \cos \left(2 \pi \Delta \phi_{p q}\right) \\
& V=D_{p q} \cos \left(2 \pi \Delta \phi_{p q}\right)-S_{p q} \sin \left(2 \pi \Delta \phi_{p q}\right) \\
& A=2 \pi \sum_{p<q} \Delta \hat{f}_{p q}{ }^{2} U, B=2 \pi \sum_{p<q} \Delta \hat{f}_{p q} \Delta \hat{g}_{p q} U \\
& C=\sum_{p<q} \Delta \hat{f}_{p q} V, D=2 \pi \sum_{p<q} \Delta \hat{g}_{p q}{ }^{2} U, E=\sum_{p<q} \Delta \hat{g}_{p q} V
\end{aligned}
$$

By Cramer's rule, 


$$
\Delta \hat{x}_{c}=\frac{C D-B E}{A D-B^{2}}, \Delta \hat{y}_{c}=\frac{A E-B C}{A D-B^{2}}
$$

\section{RESULTS AND ANALYSIS}

To test the validity of Eqs. (7), (11) and (16), two kinds of masks have been used: alternating PSM and contact array.

\section{A. Testing Eq. (7)}

An alternating PSM with $\mathrm{CD}=0.3(\lambda \mathrm{NA})$ and pitch $=10(\lambda / \mathrm{NA})$ was used for testing. Here $\lambda=248 \mathrm{~nm}$ and NA $=0.68$.

$$
\longrightarrow \text { - Theory } . . . \Delta . . \text { Aerial Image }
$$

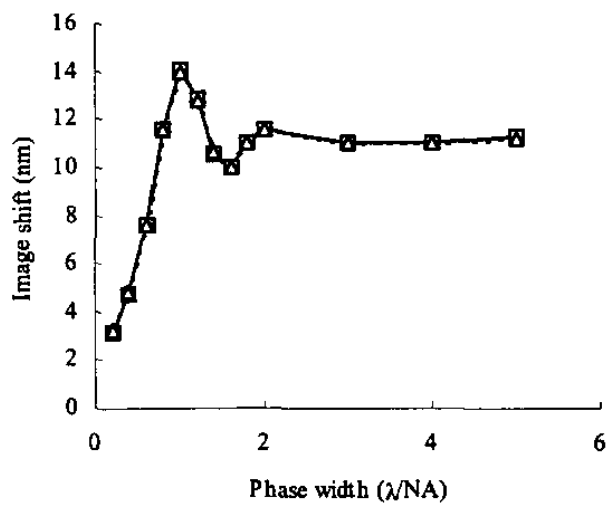

Fig. 1. Comparison between $\Delta \hat{x}_{c}$ computed from Eq. (7) and that from aerial image simulation.

Fig. 1 shows the image shift as a function of the phase width under balanced third-order coma $\Phi(\hat{\rho}, \theta)=A_{c} \sqrt{8}\left(3 \hat{\rho}^{3}-2 \hat{\rho}\right) \cos \theta$. Zernike coefficient $A_{c}=$ $0.01 \lambda$.

The two graphs show excellent agreement with each other. The average percentage error between the two sets of data is only $0.665 \%$.

\section{B. Testing Eq. (II)}

The conditions used for testing this equation are the same as those used for Eq. (7). The partial coherence factor $\sigma$ was varied. Fig. 2 and 3 show the image shift as a function of phase width when $\sigma=0.5$ and $\sigma=0.8$.

From the two figures, we can see that the two graphs show excellent agreement when $\sigma=0.5$ and $\sigma=0.8$. The average percentage errors for the two cases are only $0.519 \%$ and $0.551 \%$.

On the other hand, by observing Fig. 4, the magnitude of image shift for partially coherent imaging is, on average, smaller than that of coherent imaging.
Moreover, the oscillating behaviour of image shift at large phase width tends to be stabilized when $\sigma$ becomes higher.

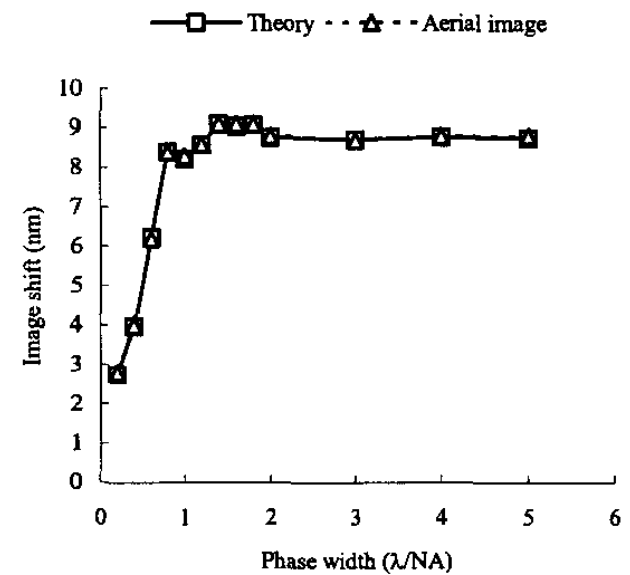

Fig. 2. Comparison between $\Delta \hat{x}_{c}$ computed from Eq. (11) and that from aerial image simulation when $\sigma=0.5$.

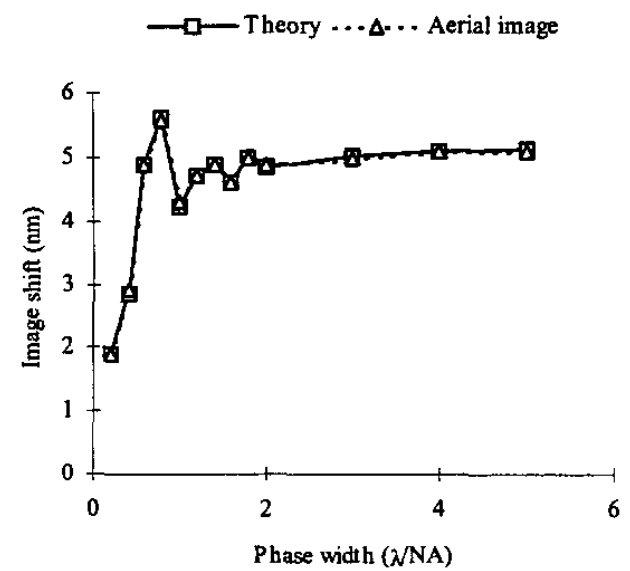

Fig. 3. Comparison between $\Delta \hat{x}_{c}$ computed from Eq. (16) and that from aerial image simulation when $\sigma=0.8$. 


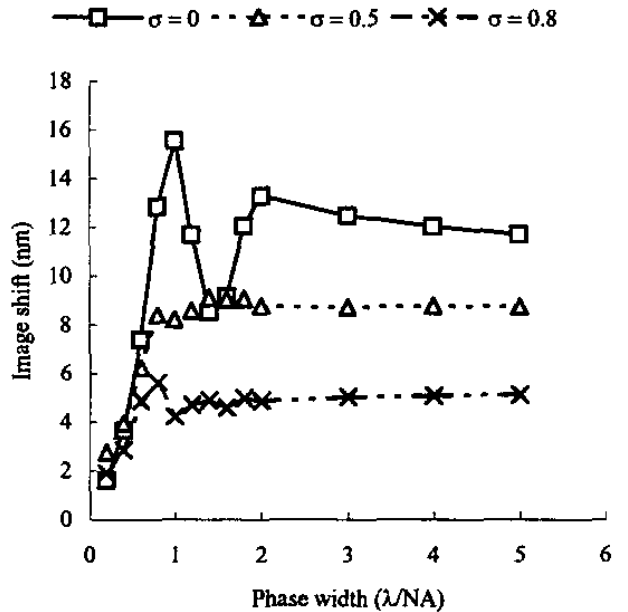

Fig. 4. Image shift decreases as partial coherence factor rises

\section{Testing Eq. (16)}

Contact array is the candidate for testing Eq. (16). The contact array being used has a pitch of $10(\lambda / \mathrm{NA})$ in both $x$ and $y$ directions. The dimensions of each contact are $0.3(\lambda / \mathrm{NA}) \times 0.3$ ( $\lambda$ /NA). During testing, the Zernike coefficient was allowed to vary from $0.001 \lambda$ to $0.22 \lambda . \lambda=$ $500 \mathrm{~nm}$ and $\mathrm{NA}=0.5$.

Fig.5 plots the image shift from Eq. (16) and that from aerial image simulation. It also shows the graph of Strehl ratio computed with Marechál formula [7] as a function of Zemike coefficient. Strehl ratio is defined as the ratio between the light intensity at the center of an image with aberration and that without aberration. According to Marech á l formula, Strehl ratio is approximately given by:

$$
S=\left(1-\sigma_{\phi}^{2} / 2\right)^{2}
$$

where $\sigma_{\phi}$ is the standard deviation of the phase aberration over the exit pupil. At the bound of validity of Eq. (16), the Strehl ratio is 0.968 . This corresponds to a wavefront error of $0.18 \lambda$ approximately. Since the conventional limit of wavefront error in the state-of-the-art exposure systems is only $0.07 \lambda[8]$, Eq. (16) is generally applicable to these exposure systems.

\section{CONCLUSION}

Theories are developed to quantify the image placement error for 1D mask spectra under coherent imaging, 1D mask spectrums under partially coherent imaging and 2D mask spectrums under coherent imaging. The validity of the equations is verified by using alternating PSM and contact array. From the graphs, it is observed that the aberration sensitivity of partially coherent imaging is lower than that of coherent imaging. In general, the theories are applicable to all types of mask features and illumination.

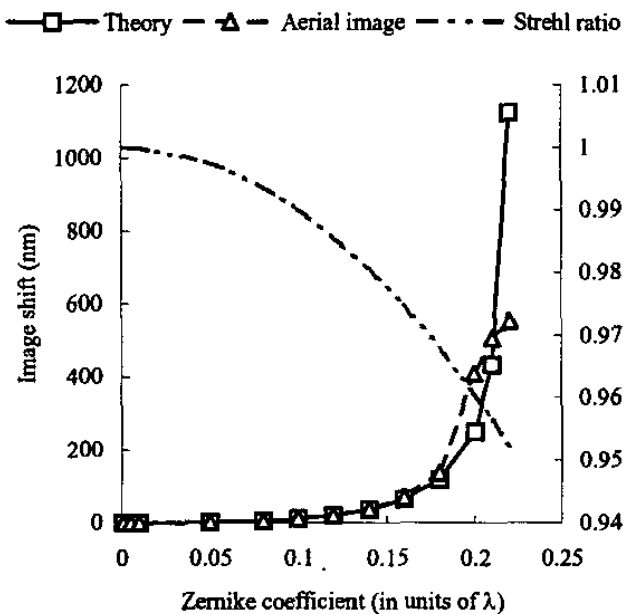

Fig. 5. Comparison between the image shift calculated from Eq. (15) and that from aerial image simulation with varying Zernike coefficient

\section{REFERENCES}

[1] D. Yim, et al., "Aberration effects in the region of 0.18 $\mu \mathrm{m}$.lithography with $\mathrm{KrF}$ excimer stepper," in Proc. $S P I E$, vol. 3051, Gene E. Fuller, ed., pp. 714-723, March 1997.

[2] A. Wong, "Theoretical discussion on reduced aberration sensitivity of enhanced alternating phase-shifting masks," in Proc. SPIE, vol. 4691, Anthony Yen, ed., pp. 359-368, 2002.

[3] S. Nakao, et al., "Impact of spherical aberrations on printing characteristics of irregularly aligned pattems of alternating phase shift mask," in Proc. SPIE, vol. 3051, pp. 672-685, March 1997.

[4] T. Brunner, "Impact of lens aberrations on optical lithography, " in IBM J. Res. Develop., vol. 41, no. 1/2, pp. 57-67, Jan. 1997.

[5] A. Wong, Resolution Enhancement Techniques in Optical Lithography, section 2.1.1, pp. 31-34. SPIE Press, 2001.

[6] A. Wong, Resolution Enhancement Techniques in Optical Lithography, section 2.3, pp. 45-54. SPIE Press, 2001.

[7] V. Mahajan, Aberration Theory Made Simple, section 8.3.1, p. 76. SPIE Press, 1998.

[8] C. Progler and D. Wheeler, "Optical lens specifications from the user's perspective," Proc. SPIE, vol. 3334, pp.256-268, June 1998. 